\title{
Prediction of sustained harmonic walking in the free- living environment using raw accelerometry data
}

Citation for published version (APA):

Urbanek, J. K., Zipunnikov, V., Harris, T., Fadel, W., Glynn, N., Koster, A., Caserotti, P., Crainiceanu, C., \& Harezlak, J. (2018). Prediction of sustained harmonic walking in the free-living environment using raw accelerometry data. Physiological Measurement, 39(2), [02NT02]. https://doi.org/10.1088/1361$6579 / a a a 74 d$

Document status and date:

Published: 01/02/2018

DOI:

10.1088/1361-6579/aaa74d

Document Version:

Publisher's PDF, also known as Version of record

Document license:

Taverne

Please check the document version of this publication:

- A submitted manuscript is the version of the article upon submission and before peer-review. There can be important differences between the submitted version and the official published version of record.

People interested in the research are advised to contact the author for the final version of the publication, or visit the DOI to the publisher's website.

- The final author version and the galley proof are versions of the publication after peer review.

- The final published version features the final layout of the paper including the volume, issue and page numbers.

Link to publication

\footnotetext{
General rights rights.

- You may freely distribute the URL identifying the publication in the public portal. please follow below link for the End User Agreement:

www.umlib.nl/taverne-license

Take down policy

If you believe that this document breaches copyright please contact us at:

repository@maastrichtuniversity.nl

providing details and we will investigate your claim.
}

Copyright and moral rights for the publications made accessible in the public portal are retained by the authors and/or other copyright owners and it is a condition of accessing publications that users recognise and abide by the legal requirements associated with these

- Users may download and print one copy of any publication from the public portal for the purpose of private study or research.

- You may not further distribute the material or use it for any profit-making activity or commercial gain

If the publication is distributed under the terms of Article $25 \mathrm{fa}$ of the Dutch Copyright Act, indicated by the "Taverne" license above, 


\title{
NOTE
}

CrossMark

\section{RECEIVED}

18 March 2017

REVISED

2 January 2018

ACCEPTED FOR PUBLICATION

12 January 2018

PUBLISHED

28 February 2018

\section{Prediction of sustained harmonic walking in the free-living environment using raw accelerometry data}

\author{
Jacek K Urbanek ${ }^{1}$, Vadim Zipunnikov ${ }^{2}$, Tamara Harris ${ }^{3}$, William Fadel ${ }^{4}$, Nancy Glynn $^{5}$, Annemarie Koster ${ }^{6}$, \\ Paolo Caserotti ${ }^{7}$, Ciprian Crainiceanu ${ }^{2}$ and Jaroslaw Harezlak ${ }^{8}$ \\ 1 Division of Geriatric Medicine and Gerontology, Department of Medicine, School of Medicine, Johns Hopkins University, \\ Baltimore, MD, United States of America \\ 2 Department of Biostatistics, Bloomberg School of Public Health, Johns Hopkins University, Baltimore, MD, United States of America \\ 3 Laboratory of Epidemiology, Demography, and Biometry, National Institute on Aging, Bethesda, MD, United States of America \\ 4 Department of Biostatistics, Indiana University School of Medicine, Indianapolis, IN, United States of America \\ 5 Center for Aging and Population Health, Department of Epidemiology, Graduate School of Public Health, University of Pittsburgh, \\ Pittsburgh, PA, United States of America \\ 6 Department of Health, Medicine and Life Sciences, Social Medicine, Maastricht University, Maastricht, Netherlands \\ 7 Department of Sports Science and Clinical Biomechanics, University of Southern Denmark, Odense, Denmark \\ 8 Department of Epidemiology and Biostatistics, Indiana University School of Public Health, Bloomington, IN, United States of America
}

E-mail:harezlak@iu.edu

Keywords: accelerometry, movement recognition, physical activity, walking quantification, wearable computing, free-living data

\begin{abstract}
Objective: Using raw, sub-second-level accelerometry data, we propose and validate a method for identifying and characterizing walking in the free-living environment. We focus on sustained harmonic walking (SHW), which we define as walking for at least $10 \mathrm{~s}$ with low variability of step frequency. Approach: We utilize the harmonic nature of SHW and quantify the local periodicity of the tri-axial raw accelerometry data. We also estimate the fundamental frequency of the observed signals and link it to the instantaneous walking (step-to-step) frequency (IWF). Next, we report the total time spent in SHW, number and durations of SHW bouts, time of the day when SHW occurred, and IWF for 49 healthy, elderly individuals. Main results: The sensitivity of the proposed classification method was found to be $97 \%$, while specificity ranged between $87 \%$ and $97 \%$ and the prediction accuracy ranged between $94 \%$ and $97 \%$. We report the total time in SHW between 140 and $10 \mathrm{~min}^{-1}$ distributed between 340 and 50 bouts. We estimate the average IWF to be 1.7 stepsper-second. Significance: We propose a simple approach for the detection of SHW and estimation of IWF, based on Fourier decomposition.
\end{abstract}

\section{Introduction}

Accelerometers are now widely used to monitor physical activity in large observational studies where thousands of subjects are observed for weeks at a time. Walking patterns are very often the main focus of such studies as they have been shown to be associated with major health and aging outcomes, including survival (Studenski et al 2011), Parkinson's disease (Din et al 2016), obesity (Browning 2006), and overall physical capability (Godfrey et al 2015). Here, we propose a method for estimating when and how people walk based on high-frequency data obtained from wearable accelerometers. These data exhibit extraordinary levels of heterogeneity due to the natural within- and between-person variability, as well as due to measuring devices that are prone to batch effects, rotations, and random artifacts. Heterogeneity makes walking prediction in the natural environment much more difficult than for 'in-the-lab' experiments (Grant et al 2006a, Ermes et al 2008, Francois et al 2009). The differences between data collected 'in-the-lab' under strict protocols and data collected in large observational studies is dramatic. Thus, we use the term data collected 'in-the-wild' to emphasize the highly unstructured nature of the data obtained during free-living human activity.

There are a number of accelerometry-based wearable devices designed to recognize and quantify walking 'in-the-wild'. These include ankle-worn step counters (Coleman et al 1999) that provide well-validated information on the occurrence and duration of walking as well as the number of steps per minute. Such devices have 
been widely used in large epidemiological studies, allowing researchers to study the walking habits of individuals (Orendurff et al 2008, Dall et al 2015). Another popular device that has been used to detect the walking patterns and posture of individuals is a thigh-worn activPAL monitor (PAL Technologies Ltd, Glasgow, UK). Grant et al (2006) discussed the validity of these devices in posture and activity recognition for 'in-the-lab' experiments, whereas in Dall et al (2015) and Klenk et al (2011) the authors use activPAL to collect data on walking patterns in a large group of participants 'in-the-wild'. While the utility of such devices is undisputable, researchers might often want to address overall physical activity levels rather than the ambulation exclusively (Weuve et al 2004). In this paper we propose a method for the detection of walking on data collected by hip-worn, accelerometry-based physical activity monitors.

Our work is preceded by a number of approaches. For example, Dijkstra et al (2008) and Weiss et al (2013) both proposed walking-recognition procedures for tri-axial, hip-worn accelerometers. These methods used a pre-defined threshold of recorded signals to classify walking activities. Lugade et al (2014) introduced an algorithm for the classification of postural orientation and movement using data collected by four custom-build activity monitors, while in their follow-up work Fortune et al (2014) discussed the problem of gait features extraction. Maurer et al (2006) presented a range of classifiers, for different body location of the sensor, including hip-worn devices. Most recently, Hickey et al (2017) introduced a prediction algorithm dedicated for data collected in the free-living environment and validated it against video recordings of daily activities.

The definition of walking based on accelerometry measurements is quite ambiguous. For example, the accelerometry signal might have a fundamentally different structure for a 6 min relaxed walk versus multiple bouts of a few seconds of walking interspersed with other activities. Moreover, laboratory studies typically collect labeled data that is consistent with 6 min of walking (e.g. a $400 \mathrm{~m}$ walk (Chang et al 2004) or simply, a 6 min walking task (Enright 2003)). Thus, in practice it is often difficult to validate walking periods that are not consistent with the laboratory-standardized definition without proper gold-standard labels (e.g. video recordings). For these reasons we focus here on sustained harmonic walking (SHW), defined as walking for at least $10 \mathrm{~s}$ with low variability of step frequency. Based on our observation, we assume that such type of walking is similar in nature to well-controlled walking, and therefore can be identified with methods trained on data collected 'in-the-lab' and applied in 'in-the-wild' settings.

\section{Methods}

\subsection{Data collection}

The data were collected in the Developmental Epidemiologic Cohort Study (DECOS), a study of healthy older individuals. The participants wore tri-axial ActiGraph GT3X+ devices that collected the raw accelerometry signal with a sampling frequency of $80 \mathrm{~Hz}$. The devices were placed on the left hip via an elastic strap. The weight of the devices was $18 \mathrm{~g}$ and the dimensions were $4.6 \times 3.3 \times 1.5 \mathrm{~cm}$.

We concentrate on the data from $N=49$ individuals ( 24 females and 25 males) who have both 'in-the-lab' and 'in-the-wild' accelerometry measurements together with associated demographic and clinical covariates. The median age of the participants was equal to 78.0 years $(\mathrm{Q} 1=74.0, \mathrm{Q} 3=82.25)$.

During the 'in-the-lab' phase of the experiment all participants were asked to perform a series of physical tasks including $400 \mathrm{~m}$ of fast walking, simulated dressing, simulated shopping, and chair stands. During the $400 \mathrm{~m}$ of fast walking the participants were instructed to walk 10 laps, of $40 \mathrm{~m}$ each, at a fast pace. For a simulated dressing activity, the participants were instructed to unfold a lab jacket, put the jacket on (no zipping or buttoning), then remove it, place it on a hanger, and put the hanger on a nearby hook. For simulated shopping participants were instructed to walk a few steps along the wall, stop by target items, remove them from the upper shelf, and place them on the lower shelf. When completed, the participants walked back in the opposite direction, moving the target items back to the top shelf.

Median time to finish a $400 \mathrm{~m}$ walk was $5 \min 49 \mathrm{~s}(\mathrm{Q} 1=4 \mathrm{~min} 40 \mathrm{~s}, \mathrm{Q} 3=6 \mathrm{~min})$. The dressing activity was set up to last exactly $3 \mathrm{~min}$, the median time to finish the shopping activity was $2 \mathrm{~min} 38 \mathrm{~s}$ (Q1 = $1 \mathrm{~min} 56 \mathrm{~s}$, $\mathrm{Q} 3=3 \mathrm{~min}$ ), and the median time of standing up from the chair task was $19 \mathrm{~s}(\mathrm{Q} 1=13 \mathrm{~s}, \mathrm{Q} 3=24 \mathrm{~s})$.

'In-the-wild' accelerometry data activities were collected over a 1-week period in the natural living environment with no activity labels.

\subsection{Modeling approach}

\subsubsection{Definitions and notation}

We define SHW as steady-pace walking performed for at least $10 \mathrm{~s}$ with a roughly constant step-to-step frequency. Here we propose a method for automatic recognition of SHW intervals in raw accelerometry data together with estimation of instantaneous walking (step-to-step) frequency. The reported frequency of human steps 'in-the-wild' ranges between 1.4-2.5 Hz (Pachi and Ji 2005). However, we will conservatively focus on the range of 1.2-4.0 Hz to include slow walking of older individuals and running. 

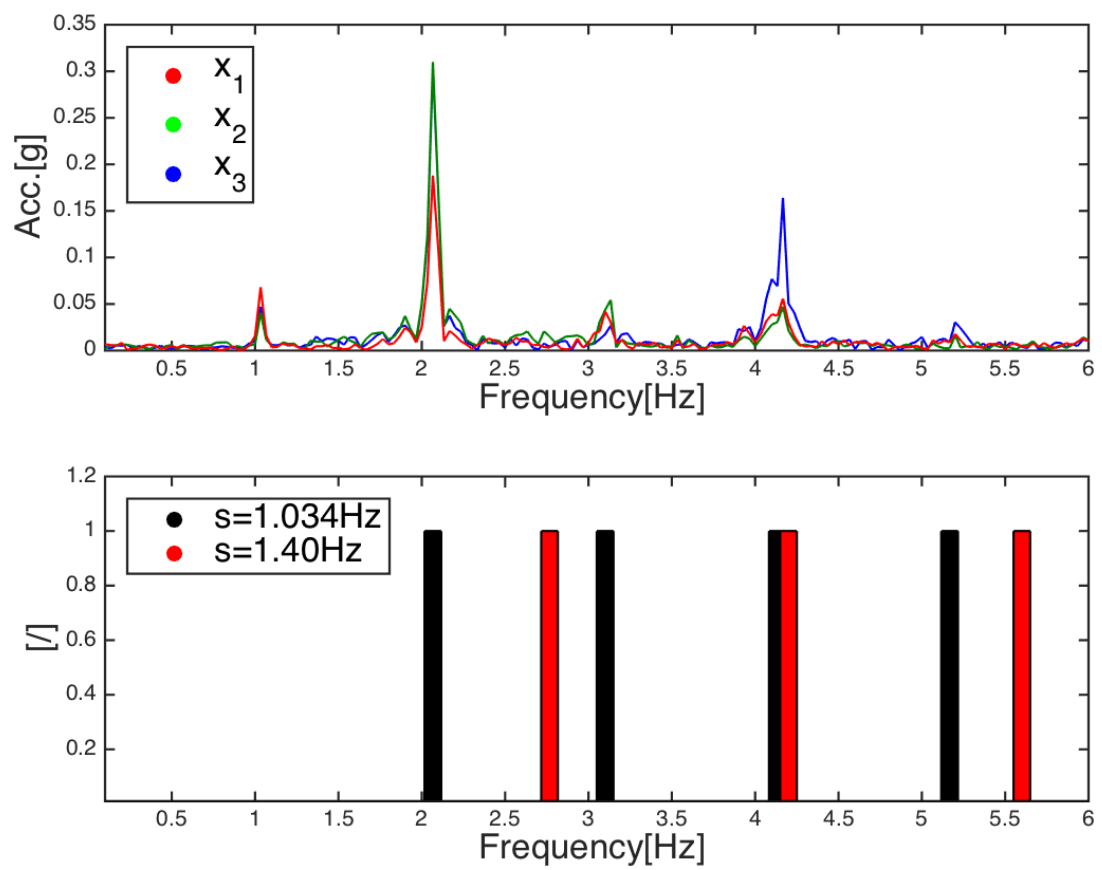

Figure 1. Top figure shows the Fourier spectra of the tri-axial acceleration signal of walking. Bottom figure shows the 'comb' function $C(f ; s)$ for $s=w(t)=1.034 \mathrm{~Hz}$ (black) and for $s=1.40 \mathrm{~Hz}$ (red). The black lines correspond to the spectral lines representing walking signal components while the red lines miss those spectral lines completely.

Let $\mathbf{x}(t)=\left\{x_{1}(t), x_{2}(t), x_{3}(t)\right\}$ denote the measured signal, where $x_{k}(t)$ (expressed in g-units) is the measurement at time index $t=1, \ldots T$ along the axis $k=1,2,3$. For notational simplicity we drop the subject index. We denote the sampling frequency of the data expressed as the number of observations per second by $f_{0}$.

Our goal is to estimate the walking indicator $y(t)$, where $y(t)=1$ corresponds to SHW and $y(t)=0$ corresponds to non-SHW. This is achieved by employing a short-time Fourier transform (STFT) for each axis separately. The discrete Fourier transform (DFT) for axis $k$ is $X_{k}(f)=\sum_{T-1}^{t=0} x_{k}(t) \mathrm{e}^{-\mathrm{i} 2 \pi f \frac{t}{T}}$, where $f$ is the frequency index, $t$ is the time index, and $T$ is the total number of observations. We define the STFT at time $t$ for axis $k$ of the acceleration signal $\mathbf{x}(t)$ as

$$
X_{k}(t, f ; \tau)=\sum_{u=[t-\tau / 2]}^{[t+\tau / 2]} x_{k}(u) h(u) \mathrm{e}^{-\mathrm{i} 2 \pi f \frac{u}{\tau}},
$$

where $\tau$ is the parameter specifying the number of observations in the interval centered at $t$. We use the Hann window defined as $h(u ; \tau)=0.5[1-\cos \{2 \pi u /(\tau-1)\}]$. The spectrum is defined as $S_{k}(t, f ; \tau)=\left|X_{k}(t, f ; \tau)\right|$, where $|\cdot|$ denotes the absolute value of a complex number. Next, we introduce the comb function, a function that 'combs' the spectrum for those frequencies that are likely to be related to walking (see figure 1). A comb function is defined by the fundamental frequency $s$, and the thickness of the comb 'teeth' with the same width for all frequencies and the total number of teeth is equal to $n_{m}$. For any frequency $s$, we define a neighborhood, $N_{s}=\left\{s-\frac{1}{U}, s, s+\frac{1}{U}\right\}$, where the local FFT is applied, where $U$ is the duration of the window expressed in seconds. Thus, $N_{s}$ is the shortest frequency interval centered at $s$ and consists of three subsequent frequency components. The comb family of functions, $C(f ; s)$, indexed by $s$, is defined as

$$
C(f ; s)=\left\{\begin{array}{l}
1 \quad \text { for } f \in \cup_{l=2}^{n_{m}} N_{l s} . \\
0
\end{array}\right.
$$

Spectra shown in the top panel of figure 1 correspond to a sample walking interval. The bottom panel displays the comb function corresponding to the fundamental frequency of the walking acceleration signal $s=1.034 \mathrm{~Hz}$. The tooth centered at $2 s=2.067 \mathrm{~Hz}$ also contains the frequencies between $1.967 \mathrm{and} 2.167 \mathrm{~Hz}$. There are additional 'comb teeth' centered at $3 s=3.102 \mathrm{~Hz}$ and so on. Figure 1 also displays an example of another comb shown in red corresponding to $s=1.40 \mathrm{~Hz}$, a frequency unrelated to a walking acceleration signal. Note that integrating the spectrum in areas corresponding to the 'black' comb will result in a higher value than for the spectrum integrating the areas corresponding to the 'red' comb, which does not match the spectral peaks. The comb function idea was inspired by the widely used comb filter of harmonic components (Chu et al 1984). However, in contrast to a filter that uses the entire range of spectral components, our comb uses only frequencies 
between $2 s$ and $n_{m} s$ to limit the number of high and low frequency components and reduce both high-frequency random noise and low-frequency oscillation resulting from other non-harmonic body movements.

\subsubsection{Prediction of walking periods and its characteristics}

We now provide the technical description of the SHW prediction approach. Specifically, for each axis $k$, we define the area under the full spectrum, $S_{k}(\cdot)$, as $I S_{k}(t)=\int_{f=0}^{f_{0} / 2} S_{k}(f, t ; \tau) \mathrm{d} f$ and the area under the spectrum corresponding to the comb function $C(f, s)$ as $I S_{k}(t, s)=\int_{f=0}^{\bar{s}_{s} / 2}(f, t ; \tau) C(f ; s) \mathrm{d} f$. All the functions under the integral are positive and $I S_{k}(t)>I S_{k}(t, s)$ for every $s$ and $t$. Next, we define

$$
Y_{k}(t, s)=\frac{I S_{k}(t, s)}{I S_{k}(t)-I S_{k}(t, s)},
$$

which is a measure of the size of the periodic content of the component of the accelerometry signal corresponding to the fundamental frequency $s$, along axis $k$.

We use $Y(t, s)=\max _{k}\left\{Y_{k}(t, s)\right\}$, which is the maximum of the fraction of the signal explained by the frequency $s$ along the three axes $k=1,2,3$ to estimate the SHW periods. For a threshold $\delta$ of $Y(t, s)$, we estimate that a person performs SHW by

$$
\widehat{y}_{\delta}(u)=\left\{\begin{array}{cc}
\text { if } \max _{s \in D_{f}} Y(t, s)>\delta, \\
0,
\end{array}\right.
$$

for every $u \in[t-\tau / 2, t+\tau / 2]$, where $\delta$ is a threshold $\delta$ of $Y(t, s)$, and $D_{f}$ is the family of frequencies corresponding to walking (here, $D_{f}=1.2, \ldots, 4 \mathrm{~Hz}$ ). Therefore, if walking was detected for only one window, it was classified as a bout lasting for $10 \mathrm{~s}$.

For the SHW periods, we estimate IWF, denoted as $\widehat{w}(t)$, as the double of frequency $s$, that maximizes $Y(t, s)$. More precisely,

$$
\widehat{w}(t)=\left\{\begin{array}{c}
2 \operatorname{argmax}_{s \in D_{f}}\{Y(t, s)\} \text { for } \widehat{y}_{\delta}(t)=1, \\
\text { Nan. }
\end{array}\right.
$$

A complete description of the approach is summarized in Algorithm 1.

Algorithm 1

Input: $\boldsymbol{x}(t)$-accelerometry signal, $f_{s}$-sampling frequency, $T$-observation time, $\tau$ - time window, $\delta$ - threshold, $s_{\min }=0.6 \mathrm{~Hz}$, $s_{\max }=2.0 \mathrm{~Hz}$.

Output: $y(t)$ _ walking indicator, ${ }^{v}(t)$ _ vector magnitude, ${ }^{w}(t)$ _IWF.

Step 1. Compute the value of the 'comb' function $C(f ; s)$ for each value of $s \in D_{f}$.

Step 2. Compute the spectrum, $S_{k}(t, f, \tau)$, for each axis $k=1,2,3$ and the area under the spectrum $I S_{k}(t)$ for each $t$.

Step 3. Compute the partial area under the spectrum $I S_{k}(t, s)$ for each $s$ and each $k$

Step 4. Calculate the periodic content of the signal $Y_{k}(t, s)=\frac{I S_{k}(t, s)}{I S_{k}(t)-I S_{k}(t, s)}$ for each $k$.

Step 5. Estimate $y_{\delta}(t)$ with $\max _{s \in D_{f}} Y_{k}(t, s)$.

Step 6. For the times $t$ with $\widehat{y}_{\delta}(t)=1$ estimate $w(t)$ by finding the $s$ that maximizes $Y(t, s)$.

Step 7. Identify walking if any of the estimators $\widehat{y}_{\delta}(t)$ is 1 .

\subsection{Validation using the 'in-the-lab' data}

To validate the method, we used data collected during the 'in-the-lab' phase of the DECOS study.

\subsubsection{Selection of the tuning parameters}

In this section, we discuss the tuning parameter choices: the window length $\tau$, the threshold $\delta$ of $Y(t, s)$, and the number of harmonics (comb teeth) $n_{m}$.

There are trade-offs in choosing $\tau$ as (1) longer windows result in more precise spectrum estimation, while (2) shorter time windows are less likely to capture changes in walking frequency or changes in activity type. Based on the empirical evaluation of the 'in-the-lab' data, we found that a $\tau=10 \mathrm{~s}$ interval is long enough for people to maintain their IWF yet short enough to not be sensitive to smooth changes in IWF, resulting in clean spectral signatures.

SHW is estimated in time increments equal to $1 \mathrm{~s}$, so consecutive windows overlap by $90 \%$ and a $1 \mathrm{~s}$ interval is declared to be SHW if any of the intervals containing it is estimated to be a SHW. The duration of each walking bout is equal to the number of consecutive time windows where walking was estimated to have occurred plus the window duration multiplied by the overlap. For example, if walking was detected in 8 consecutive windows, the 


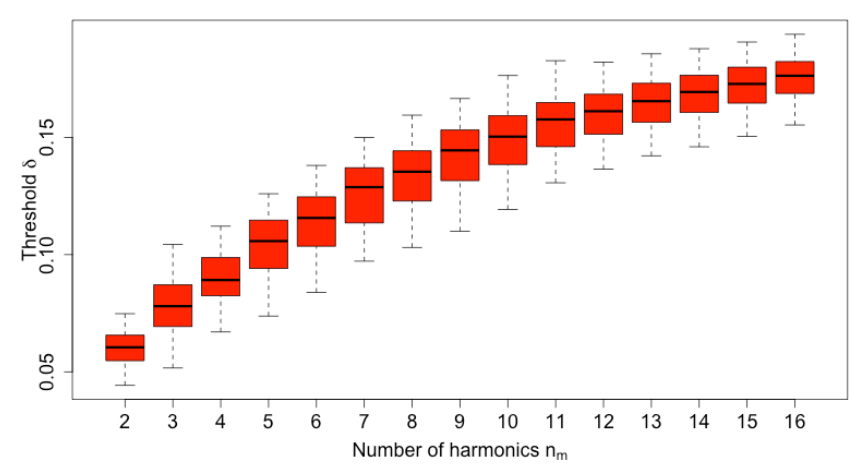

Figure 2. Boxplot representing distributions of subject-specific $\delta$ versus $n_{m}$

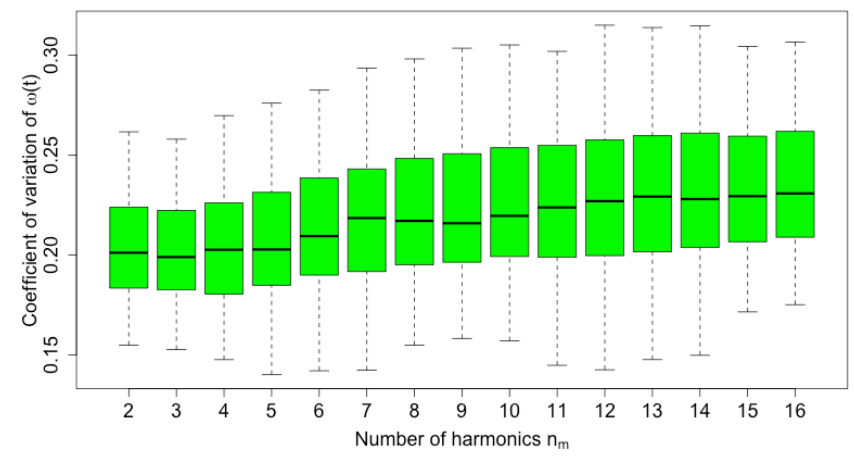

Figure 3. Boxplot representing coefficient of variation as a function of the number of harmonics.

walking bout was determined to have lasted $8+0.9 \times 10=17 \mathrm{~s}$. It is important to note that we cannot estimate a length of a walking bout that is shorter than the length of the window $\tau$. Therefore, if walking was detected for only one window, it was classified as a bout lasting for $10 \mathrm{~s}$.

The choice of the threshold $\delta$ and the number of harmonics $n_{m}$ are inter-related. The proportion of the variability $\left(\max _{s \in D_{f}} Y(t, s)\right)$ explained is an increasing function of the number of harmonics $n_{m}$. We studied $\delta$ as a function of $n_{m}$, for $n_{m}=2, \ldots, 17$, where the upper limit of 17 is determined by the sampling frequency of the raw accelerometry data. We estimate the density function of $\max _{s \in D_{f}} Y(t, s)$ for all SHW and non-SHW periods for all subjects. The parameter $\delta$ was then estimated for each subject separately as the intersection between the subject-specific SHW and non-SHW density functions. Figure 2 displays a boxplot of the estimated $\delta$ values across subjects as a function of the number of harmonics, $n_{m}$. While some between-subject variability exists in the estimation of $\delta$ at every value of $n_{m}$, having a population level value simplifies the procedure considerably. For example, when $n_{m}=6$ harmonics are used as an estimated median value of $\delta$ if the population level is equal to 0.115 .

The selection of the number of harmonics, $n_{m}$, is important for the estimation of IWF, $w(t)$ (equation (5)). In principle, we would like to utilize as many frequencies as possible without degrading the IWF estimation. To study the choice of $n_{m}$, we calculated the IWF, $w(t)$, for every subject at each time point during their $400 \mathrm{~m}$ walk. For every subject, we then calculated the coefficient of variation of the IWF during this period. Given that this is a well-controlled experiment, the coefficient of variation of the IWF is expected to be small. Figure 3 displays the coefficient of variation as a function of the number of harmonics for every subject. The average coefficient of variation is relatively stable for $n_{m}=2, \ldots, 6$ and it starts to increase for larger values of $n_{m}$. Thus, to use as much information as possible and still keep the coefficient of variation small, we selected $n_{m}=6$.

\subsubsection{Performance of the algorithm}

For further processing we used $\tau=10$ and $\delta=0.115$ and a range of possible IWFs between 1.2 and $4.0 \mathrm{steps} \mathrm{s}^{-1}$. The upper boundary for possible IWFs was purposefully set to be relatively high to account for running for active individuals.

Next, we calculated the specificity, sensitivity, and prediction accuracy of the algorithm in three separate classification tasks. Namely, shopping versus walking, chair stands versus walking, and dressing versus walking. Shopping, chair stands, and dressing were defined as non-walking activities, whereas a $400 \mathrm{~m}$ walk was defined as a walking activity. For shopping and chair standing activities specificity was equal to $95 \%$ and $97 \%$, respectively. 

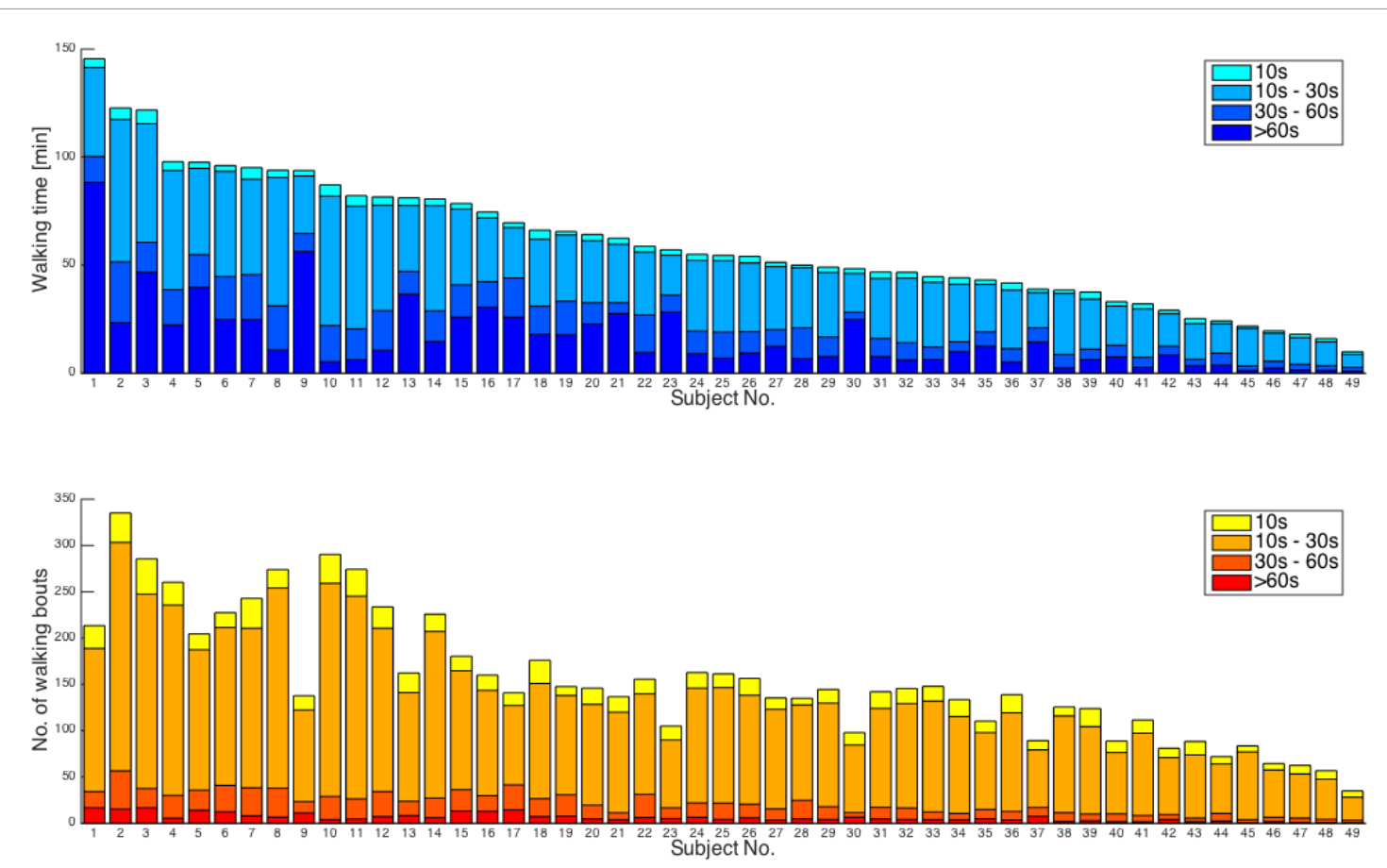

Figure 4. Bar plot presenting the total time of walking and corresponding number of bouts.

Specificity was the lowest for the dressing activity and was equal to $87 \%$. The sensitivity of the classifier was equal to $97 \%$. The prediction accuracy was equal to $97 \%$ for shopping versus walking and chair stands versus walking and $94 \%$ for dressing versus walking.

\section{Analysis of 'in-the-wild' data}

We applied the approach described in section 2.3 to data collected during the 'in-the-wild' portion of the experiment, to estimate when SHW occurs together with the corresponding IWF. We used the same tuning parameters as in section 2.3.2 $\left(\tau=10\right.$ and $\delta=0.115$ and a range of possible IWFs between 1.2 and $4.0 \mathrm{steps} \mathrm{s}^{-1}$, with a $90 \%$ time-window overlap).

Figure 4 displays the total SHW time (top panel) for each of the 49 participants together with the corresponding total number of walking bouts (bottom panel) as per-day averages from the $7 \mathrm{~d}$ of activity. The results are sorted in decreasing order according to the estimated average total walking time. Color shading corresponds to different lengths of walking bouts. For example, the width of the lightest blue bars in the top panel of figure 4 corresponds to the total walking time from walking bouts equal to $10 \mathrm{~s}$. The yellow bars in the bottom panel of figure 4 display the total number of bouts equal to $10 \mathrm{~s}$. The results indicate that a long SHW time does not necessarily indicate a large number of SHW bouts. For example, subject 1 had the longest SHW time per day (140 min), spread over 210 SHW bouts, which is in about the third quartile of the number of SHW bouts for this group. The results indicate that the majority of daily walking bouts for all subjects are between 10 and $30 \mathrm{~s}$.

Figure 5 provides a lasagna plot (Swihart et al 2010) for daily walking patterns for all subjects. The shades of blue correspond to the number of minutes of walking within a $1 \mathrm{~h}$ window. Each row corresponds to $1 \mathrm{~d}$ and the dashed red lines separate the data for individual subjects. The results were sorted using the same ordering as in figure 4, with the subjects with the highest average daily walking time shown at the top.

Figure 6 displays the boxplots of the IWF for each subject sorted according to total walking time. The width of the boxes is proportional to the number of walking bouts.

\section{Summary and discussion}

We have introduced the definition of SHW together with the classification algorithm based on the quantification of the 'local degree of periodicity' of all tri-axial time series. The performance of our method depends strongly on the assumption that SHW is a repetitive and sustained process in a particular time window. For example, it is possible for a window of $10 \mathrm{~s}$ to contain 3 to $4 \mathrm{~s}$ of walking which are not recognized as walking. Thus, our method will tend to work well at recognizing sustained walking for 10 and more seconds. This can be seen in the results obtained 'in-the-lab' where specificity for shopping versus walking was $95 \%$ even though the shopping task consisted of some non-sustained walking. The performance of the proposed method is comparable to previously 


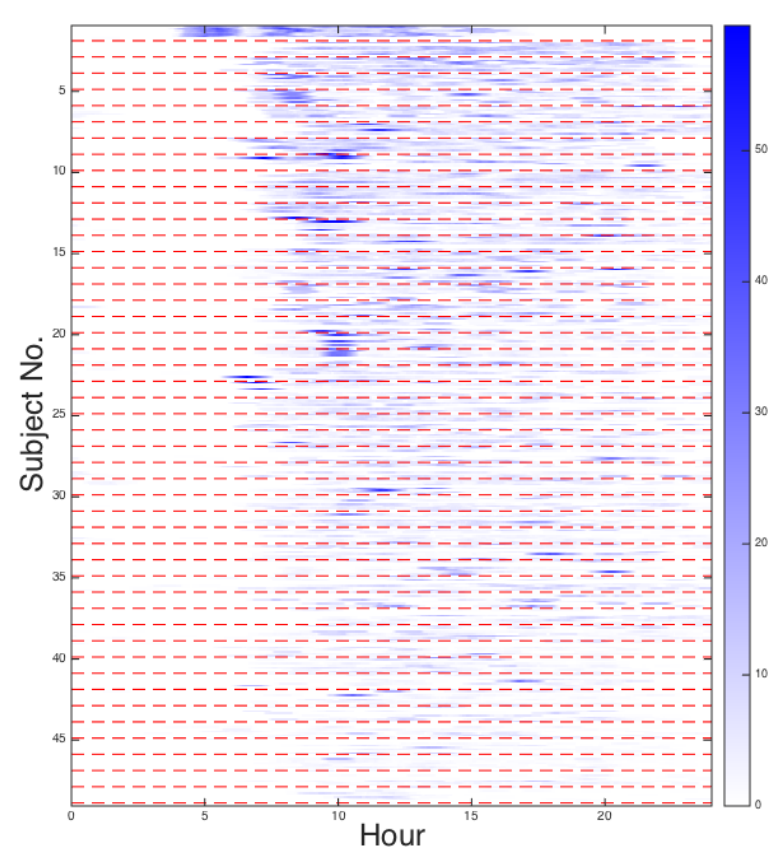

Figure 5. Lasagna plots presenting the number of minutes of walking per $1 \mathrm{~h}$. Rows represent consecutive days. Red lines separate different subjects.

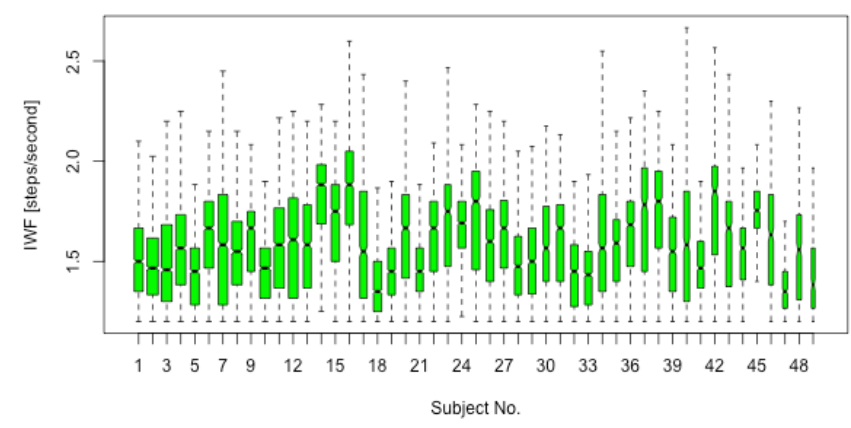

Figure 6. Box-plot presenting the IWF for each participant observed 'in-the-wild'. The width of the boxes is proportional to the number of observations.

published results. For example, Dijkstra et al (2008) reported a sensitivity of 89.5\%, whereas Lugade et al (2014) obtained a median sensitivity ranging between $84 \%$ and $95 \%$ depending on the gait velocity. Also, Maurer et al (2006) reported a prediction accuracy equal to $87 \%$ for hip worn accelerometers. That compares favorably to our approach with a prediction accuracy between $94 \%$ and $97 \%$.

Based on our interpretation of the data we assumed that SHW is similar in both 'in-the-lab' and 'in-the-wild' settings and that methods developed using 'in-the-lab' data can be scaled-up to the free-living experiment. In reality, however, it is difficult to talk about classifiers performance 'in-the-wild' without proper gold standard labels. We believe that our method performs well based on a comparison of the obtained results to previously published studies that used accelerometry. For example, Klenk et al (2011) reported a similar daily walking duration in an elderly population, equal to $104.4 \pm 50.7 \mathrm{~min}$ and $102.9 \pm 47.8 \mathrm{~min}$ for males and females, respectively. In Orendurff et al (2008), the authors presented a comparable distribution of durations of walking bouts, where $60 \%$ of all walking bouts lasted 30 s or less, with $20.1 \%$ of walking bouts lasting for 10 s or less. This suggests a significant number of walking bouts that do not fall under the definition of SHW. Dall et al (2015) reported an average value of step frequency of around 2.0 steps s $^{-1}$, therefore slightly higher than indicated by our results. A possible explanation for this discrepancy is that the studied population was much younger (mean age 46 years), and therefore characterized by a higher gait speed (Studenski et al 2011) and consequently higher step frequency. Although the arguments given above cannot replace proper validation of our algorithm's performance, they suggest that our method is promising for analyzing high-density accelerometry data collected 'in-the-wild'. 
In future studies, we will explore alternative validation methods for movement recognition 'in-the-wild' using multiple wearable sensors as well as investigating the associations between the SHW features and health outcomes.

\section{Acknowledgments}

This research was supported by the NIH under Grant No. RC2AG036594, the Pittsburgh Claude D. Pepper Older Americans Independence Center, Research Registry and Developmental Pilot under Grant Nos. NIH P30 AG024826 and NIH P30 AG024827, and the National Institute on Aging Professional Services under Contract No. HHSN271201100605P. This project was also supported, in part, by the Intramural Research Program of the National Institute on Aging. This research was supported by the NIH under Grant No. RO1 NS085211 from the National Institute of Neurological Disorders and Stroke, under NIH Grant No. RO1 MH095836 from the National Institute of Mental Health and NIH Grant No. 1R01 HL123407 from the National Heart, Lung and Blood Institute.

\section{References}

Browning R C 2006 Effects of obesity and sex on the energetic cost and preferred speed of walking J. Appl. Physiol. 100 390-8

Chang M, Cohen-Mansfield J, Ferrucci L, Leveille S, Volpato S, De Rekeneire N and Guralnik J M 2004 Incidence of loss of ability to walk 400 meters in a functionally limited older population J. Am. Geriatr. Soc. 52 2094-8

Chastin S F M and Granat M H 2010 Methods for objective measure, quantification and analysis of sedentary behaviour and inactivity Gait \& Posture 31 82-6

Chu S and Burrus C 1984 Multirate filter designs using comb filters IEEE Trans. Circuits Syst. 31 913-24

Coleman K L, Smith D G, Boone D A, Joseph A W and Aguila M A 1999 Step activity monitor: long-term, continuous recording of ambulatory function J. Rehabilitation Research and Development 36 8-18

Dall P, Robert P and McCrorie W 2015 Step accumulation per minute epoch is not the same as cadence for free-living adultsMed Sci Sports Exerc 45 1995-2001

Dijkstra B, Zijlstra W, Scherder E, Kamsma Y and British Geriatrics Society 2008 Detection of walking periods and number of steps in older adults and patients with Parkinson's disease: accuracy of a pedometer and an accelerometry-based method Age and Ageing 37 436-41

Din S D, Godfrey A, Galna B, Lord S and Rochester L 2016 Free-living gait characteristics in ageing and Parkinson's disease: impact of environment and ambulatory bout length J. Neuroeng. Rehabil. 1346

Enright P L 2003 The six-minute walk test Respir. Care 48 783-5

Ermes M, Parkka J, Mantyjarvi J and Korhonen I 2008 Detection of daily activities and sports with wearable sensors in controlled and uncontrolled conditions IEEE Trans. Inf. Technol. Biomed. 12 20-6

Fortune E, Lugade V, Morrow M and Kaufman K 2014 Validity of using tri-axial accelerometers to measure human movement-part II: step counts at a wide range of gait velocities Med. Eng. Phys.36 659-69

Godfrey A, Lara J, Munro CA, Wiuff C, Chowdhury S A, Din S D, Hickey A, Mathers J C and Rochester L 2015 Instrumented assessment of test battery for physical capability using an accelerometer: a feasibility study instrumented assessment of test battery for physical capability using an accelerometer: a feasibility study Physiol. Meas. 36 N71-N83

Grant P M, Ryan C G, Tigbe W W and Granat M H 2006 The validation of a novel activity monitor in the measurement of posture and motion during everyday activities Br. J. Sports Med. 40 992-7

Hickey A, Del Din S, Rochester L and Godfrey A 2017 Detecting free-living steps and walking bouts: validating an algorithm for macro gait analysis Physiol. Meas. $38 \mathrm{~N} 1-15$

Klenk J, Büchele G, Rapp K, Franke S, Peter R and ActiFE Study Group 2011 Walking on sunshine: effect of weather conditions on physical activity in older people J. Epidemiology \& Community Health jech-2010 (https://doi.org/10.1136/jech.2010.128090)

Lugade V, Fortune E, Morrow M and Kaufman K 2014 Validity of using tri-axial accelerometers to measure human movement-part I: posture and movement detection Med. Eng. Phys. 36 169-76

Maurer U, Smailagic A, Siewiorek D P and Deisher M 2006 Activity recognition and monitoring using multiple sensors on different body positions Int. Workshop on Wearable and Implantable Body Sensor Networks, 2006. BSN 2006 (IEEE) p 4

Orendurff M S, Schoen J A, Bernatz G C, Segal A D and Klute G K 2008 How humans walk: bout duration, steps per bout, and rest duration J. Rehabilitation Research and Development 451077

Pachi A and Ji T 2005 Frequency and velocity of people walking Struct. Eng. 83 36-40

Studenski $S$ et al 2011 Gait speed and survival in older adults JAMA 305 50-8

Swihart B J, Caffo B, James B D, Strand M, Schwartz B S and Punjabi N M 2010 Lasagna plots: a saucy alternative to spaghetti plots Epidemiology 21621

Weiss A, Brozgol M, Dorfman M, Herman T, Shema S, Giladi N and Hausdorff J M 2013 Does the evaluation of gait quality during daily life provide insight into fall risk? A novel approach using 3 day accelerometer recordings Neurorehabil. Neural Repair 27 742-52

Weuve J, Kang J H, Manson J E, Breteler M M B, Ware J H and Grodstein F 2004 Physical activity, including walking, and cognitive function in older women Jama 292 1454-61 